\title{
The central roles of exosomes in hematological malignancies: A new frontier review
}

\author{
Bing Xia ${ }^{1 *}$, Mengzhen $\mathrm{Li}^{1}$, Ruifang Yang ${ }^{2}$, Xi Wang ${ }^{1}$, Chengtao Shun ${ }^{1}$ and Yizhuo Zhang ${ }^{* 1,3}$ \\ ${ }^{1}$ Department of Hematology, Tianjin Medical University Cancer Institute and Hospital, National Clinical \\ Research Center for Cancer; Key Laboratory of Cancer Prevention and Therapy, Tianjin; Tianjin's Clinical \\ Research Center for Cancer; 300060, China \\ ${ }^{2}$ Department of Clinical Laboratory, Tianjin Medical University Cancer Institute and Hospital,National \\ Clinical Research Center for Cancer; Key Laboratory of Cancer Prevention and Therapy, Tianjin; Tianjin's \\ Clinical Research Center for Cancer; 300060, China \\ ${ }^{3}$ Department of Pediatric Oncology, Sun Yat-sen University Cancer Center, State Key Laboratory of Oncology \\ in South China, Collaborative Innovation Center for Cancer Medicine, Guangzhou, Guangdong 510060, China
}

\begin{abstract}
Exosomes, which are 30- to $120-\mathrm{nm}$ vesicles, are released by most types of cells, including tumor cells. In hematological malignancies, exosome-mediated expulsion of a number of key proteins and micro RNAs, resulting in influence of major tumor-related pathways. Emerging evidence suggests that the component secreted by exosomescan promote tumor survival, angiogenesis and metastasis, and also mediate tumor microenvironment induced drug resistance and immune escape. Furthermore, exosomes contain a great variety of bioactive molecules and are emerging as rich reservoirs of hematological tumor-specific biomarkers for the detection and therapeutics.This comprehensive review highlights the advancements in understanding of the pathogenesis of exosomes secretion and the consequence onhematological malignancies development. Full knowledge of the contribution of exosomes to the potential medical application ofdiagnosis and treatment will depend on the ingenuity of future investigators and their insight into biological processes.
\end{abstract}

KEY WORDS: HEMATOLOGICAL MALIGNANCIES; EXOSOMES; DISEASE DEVELOPMENT;DIAGNOSIS; TREATMENT

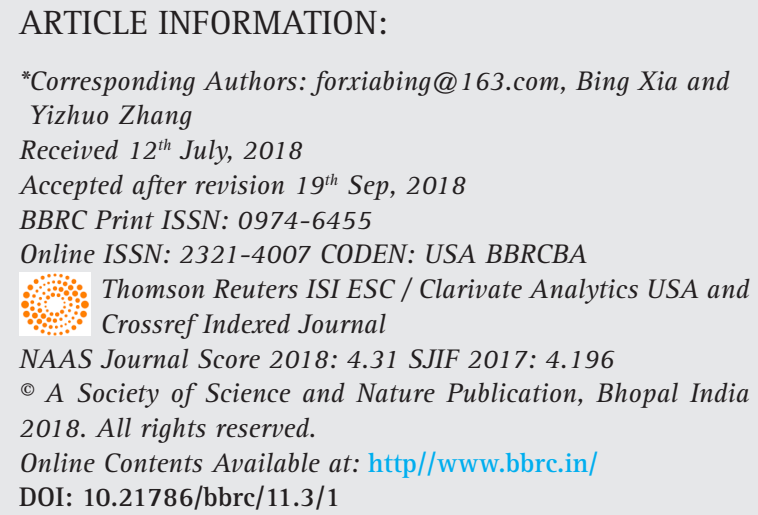




\section{INTRODUCTION}

Hematological malignancies, which include lymphoid, myeloid, histiocytic, and mast cell neoplasms, are a heterogeneous group of diseases of diverse incidence, pathogenesis and prognosis (Kornblau et al., 1998). The prognosis of hematological malignancies patients diverges greatly, largely depending on thepathological types of the patient (Li et al., 2016). Nonetheless, enhancing understanding of tumorigenesis mechanism is critical for development of novel diagnosis and therapeutic strategy. Exosomes are a kind of extracellular vesicles (EVs), which was first reported by Pan and Johnstone in 1983 as unwanted cellular components extruding from reticulocytes. EVs are medium-sized vesicles, ranging from 30 to $120 \mathrm{~nm}$ and are secreted by different cell types under both physiological and pathological conditions. Exosomes are rich in cholesterol, sphingomyelin, ceramide lipids, protein, mRNA, miRNA, and various other signaling molecules from donor cell. In addition, CD9, CD63, and CD81 are most frequently detected and are considered asthe classic markers of exosomes, (Malla et al., 2018).

Then, the MVB's can either fuse with lysosomes resulting in degradation of intra-luminal contents or they can secrete their content as exosomes outside the donor cells. Furthermore, there are several ways by which exosomes are taken up by recipient cells: receptor- or lipiddraftmediated endocytosis, phagocytosis, macropinocytosis, or fusion with the plasma membrane of a target cell, For transportation, exosomes mainly originate from multi- vesicular bodies (MVB's) in the cells which are produced by the invagination of endosomal limiting membrane (Pfrieger et al., 2018).

Recently, it has been reported by many workers that the potential functions of exosomes contributed to various aspects of hematological tumorigenesis, particularly with a focus on the exosome-mediated tumor progression, metastasis, drug resistance and immune escape by altering the function of receiver cells via diverse exosomal cargoes including proteins, DNA, messenger RNAs (mRNAs), and microRNAs) (Whiteside and Boyiadzis, (2017). Furthermore, exosomes are found in most biological fluids including urine, blood, ascites, saliva and cerebrospinal fluid, which can be made for very attractive targets for diagnostic application.Although the known information is limited, exosomes have been reported to play an emerging role in various aspects of the tumor survival, metastasis, drug resistance, and immune escape of hematologic tumor. In this review, we will focus specifically on the effects of exosomes on tumorigenesis, diagnosis and treatment of hematological malignanciesand the relevantnew prospects.

\section{THE ROLE OF EXOSOMES IN DEVELOPMENT OF HEMATOLOGICAL MALIGNANCIES}

Recently, considerable amount of studies have revealed that exosomes were secreted by tumor cells ortumor microenvironment cells and cross-talk influence tumor proliferation, angiogenesis, drug resistance and immune escape of several hematological cell types (figure1) (Sun, et al., 2018).

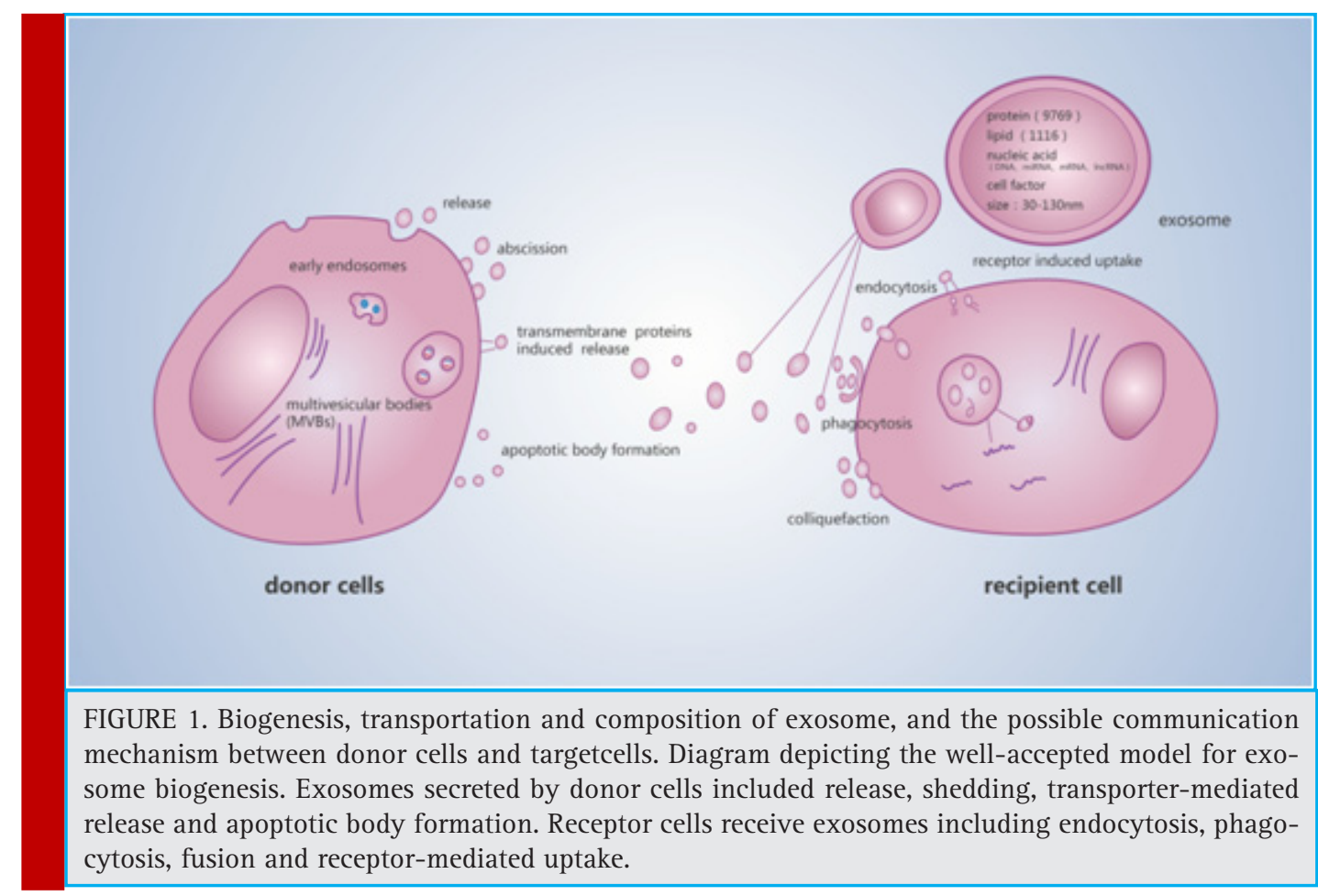




\section{Survival and proliferation}

One study demonstrated that the level of sera-derived exosomes in acute myeloid leukemia (AML) patients are higher than sera-derived exosomes from healthy controls [Hong et al., 2014] and the exosomes secreted by AML cells express higher level of mRNA, such as GATA1, F0X3, SHIP1, ID1, E2F1, CEBP- $\alpha$, CEBP- $\beta$, Myc and MEF2C, which are the group of transcripts of genes to the development of AML [Huan J. et al., 2013]. Furthermore, another study showed that levels of active TGF- $\beta 1$ carried by exosomes obtained at AML diagnosis were high and then decreased following induction chemotherapy. In addition, in long-term remission of AML patients, TGF- $\beta 1$ levels in plasma-derived exosomes approach the same levels seen in exosomes of normal controls. The results indicate that exosomal protein and TGF- $\beta 1$ levels in AML plasma could serve as biomarkers of response to chemotherapy.These data showed that both AML cell lines and primary AML blasts released exosomes relevant to AML pathogenesis (Huan et al., 2013 and Long et al., 2017).

For chronic myelocytic leukemia (CML), CML cell line LAMA84-derived exosomes increase levels of IL-8 mRNA and protein in HS-5 (bone marrow stromal cell line), and further promote HS-5 induced CML cell proliferation, (Zhou et al., 2012). In another way, the exosomes also reduce expression of the proapoptotic genes BAD, BAX and PUMA and increase expression of anti-apoptotic genes BCL-xL, BCL-w, and BIRC5.Moreover, exosomes also activate the PI3/AKT and MAPK/ERK signaling pathways. Collectively, the exosomes secreted by CML cells both educate the tumor microenvironment cells and directly affected the proliferation and apoptosis of LAMA84 cells and finally promote the CML cells survival. Human T-lymphotropic virus type 1 (HTLV1) infected $\mathrm{T}$ cells release exosomes that contain viral Tax protein and Tax, HBV, and EBV mRNA, which increase levels of phosphorylated AKT and active NF-kB pathway, and further facilitate T-cell tumor cells survival. These results suggest that the exosomes released from HTLV-1-infected cells play key role in the pathogenesis of T-cell leukemia (Jaworski et al., 2014, Raimondo et al., 2015).

The study by Raphael Koch shows that diffuse large B-cell lymphoma (DLBCL) possess a self-organized infrastructure comprising side population (SP) and non-SP cells, where transitions between clonogenic states aremodulated by exosomes mediated Wnt signaling. Lymphoma SP cells exhibit autonomous clonogenicity and export Wnt3a via exosomes to neighboring cells, thus modulating population equilibrium in the tumor (Koch et al., 2014). The study about the role in MCL observes that MCL exosomes are taken up rapidly and preferentially by MCL cells. Only a minor fraction of exosomes was internalized into T-cell leukemia and bone marrow stroma cell lines, when these cells werecocultured with MCL cells. Moreover, MCL patients' exosomes were taken up by both healthy and patients' B-lymphocytes with no apparent internalization to $\mathrm{T}$ lymphocytes and NK cells. Exosome internalization was not inhibited by specific siRNA against caveolin 1 and clathrin but was found to be mediated by a cholesterol-dependent pathway. These findings demonstrate natural specificity of exosomes to B-lymphocytes and ultimately might be used for therapeutic intervention in B cells malignancies, (Hazan et al., 2015).

EBV infection of B cells in vitro induces the release of exosomes that harbor the viral latent membrane protein 1 (LMP1). LMP1 via exosomes actives CD40 signaling and induces proliferation of B lymphocytes and $\mathrm{T}$ cell independent class-switch recombination.Finally, LMP1 drove B cell differentiation toward a plasmablast-like phenotype. In conclusion, the results suggest that exosomes released by EBV-infected lymphoma cells include the production of the activation-induced cytidine deaminase (AID), and further promote tumor cells aggressive and progression, ( Nanbo et al., 2013). In addition, Chugh et al. (2013) reported that exosomes derived from patients with KSHV-associated malignancies and KSHV mouse modelscontained KSHV encoded microRNAs such as miR-17-92 cluster, which are affect the targets of KSHV signaling pathways that may therefore be part of the paracrine signaling mechanism that mediates KSHV pathogenesis.

A research about relationship between MSC and plasma cells demonstrated that the tumor-supportiverole of BMSC-derived exosomes: Compared with normal mesenchymal stem cells, BMSC-derived exosomes in MMexpresslower level of microRNA15a and microRNA15a isa tumor-suppress factor thatcontributeto MM disease progression. In addition, BMSC-derived exosomes in MM expresshigher content levels fibronectin, indicating that BM-MSC-derived exosomes may differentially impact MM cell adhesion, (Roccaro et al., 2013). Kim De Veirman et al. (2016) showed that miRNA-146a in MMderived exosomes can be transmitted into mesenchymal stem cells, resulting in induction of expression for some cytokines and chemokines including CXCL1, IL6, IL-8, IP10, MCP-1, and CCL-5, thus lead to increasing vitality and progression of MM cells.

Angiogenesis and metastasis:Exosomes have been increasingly recognized as a new mediator for angiogenesis and metastasis of hematological malignancies. For example, Umezu et al.(2013) showed that leukemia cells K562 released the exosomal miRNAs, such as miR17-92 cluster, especially miR-92a, into human umbilical vein endothelial cells (HUVECs) , and target reduced the expression of integrin $\alpha 5$ in HUVECs by exosomal 
miR-92a, indicating that exogenous miRNA via exosomal transport can function like endogenous miRNA in HUVECs, which enhance endothelial cell migration and tube formation. In the study, which investigated the angiogenic role of exosomes produced by acute promyelocytic leukemia cells NB4.PML-RARA transcript has been detected in NB4 exosomes and taken up by endothelial cells, resulting in decreasing the levels of VEGF and tissue factor (TF) through increasing IL-8 mRNA and protein content in their EVs, renders the HUVECs more TF-positive and procoagulant, (Fang et al., 2016).

A recent study by Hiroko Tadokoro et al. has clearly demonstrated that hypoxia promotes the release of exosomes in K562 cells, the amount of exosomal miR210, which down-regulated EFNA3, an inhibitor of angiogenesis, (Tadokoro et al., 2013). The results suggest that exosomal miRNA derived from cancer cells under hypoxic conditions may partly affect angiogenic activity in endothelial cells. Paggetti et al. found that CLLderived exosomes are actively incorporated by endothelial and mesenchymal stem cells ex vivo and in vivo and that the transfer of exosomal protein and microRNA induces an inflammatory phenotype in the target cells, which resembles the phenotype of cancer-associated fibroblasts (CAFs), (Paggetti et al., 2015).

Exosomeswere uptakenby endothelial cells increased angiogenesis ex vivo and in vivo, and coinjection of CLL-derived exosomes and CLL cells promoted tumor growth in immunodeficient mice. These findings demonstrate that CLL-derived exosomes actively promote disease progression by modulating several functions of surrounding stromal cells that acquire features of cancer-associated fibroblasts, (Paggetti et al., 2015). Another similar study found that EBV-positive Burkitt's lymphoma cells Raji released exosomes with miR-155 inducing angiogenesis in remote recipient cells, whereas no major difference was found in co-culture with EBVnegative Burkitt's lymphoma cells, (Yoon et al., 2016).

Thus, it would be reasonable to believe that specific viral exosomal microRNAs contribute to angiogenesis of vascular endothelial cells, subsequently leading to pathophysiologic angiogenesis. In accord with a study by Umezu et al. (2014), miRNA-135b from MM-derived exosomes accelerated HIF-1 transcriptional activity via inhibition of FIH-1, which is called the HIF-FIH signaling pathway, exertinganangiogenesis influence. Collectively, these results suggest that hematological malignancy cells release exosomes that could promote tumor metastasis and the formation of pre-metastatic niches to create a microenvironment favorable to survival and proliferation of tumor cells themselves.

Drug resistance: Like many other tumors, increasing evidences also revealed that the tumor microenvironment
(TME) has crucial impact on hematological malignancies initiation and progression (Rizzo et al., 2011). Given the central role of exosomes in cellular communication, it is undoubtable that exosomes also contribute to microenvironment-induced drug resistance. One intuitive mechanism involving exosomes would be the sequestration of cytotoxic drugs in the intracellular vesicles and subsequent expulsion, to negate drug effect within the cells. Notably, emerging evidence suggests that both the exosomes released from TME cells and the tumor cells themselves help the hematological malignancy cells to resist chemotherapy, (Isidori et al., 2014). One prominent example, exosomes from both AML-BMSC and healthy controls protect MOLM-14 FLT3 internal tandem duplication (FLT3-ITD+) AML cells from cytarabine and stromal exosomes alter chemo-resistance in AML cells. Further, only AML-BMSC exosomes significantly protected AML cells from the FLT3 inhibitor AC220 after exposure. The protection might be associated with elevated level of miRNA-155, miRNA-375, cytokine epidermal growth factor (EGF) and TGF- $\beta 1$ (Viola et al., 2016).

Finally, these data imply a few novel approaches to overcome drug resistance on AML blasts by either blocking exosome-leukemia cell communication, or inhibiting tumor microenvironment exosome production. Moreover, several studies unveiled that galectin-3 was upregulated and stabilized anti-apoptotic Bcl-2 family proteins in survival leukemia cells, which facilitates escape from apoptotic stimuli through activation of $\mathrm{Wnt} / \beta$-Catenin signaling pathway and the PI3K/Akt pathway, (Cheng et al., 2011, Hu K. et al., 2015).

In another study about lymphoma, the results revealed that high levels of adenosine triphosphate (ATP)-binding cassette $(\mathrm{ABC})$ transporter $\mathrm{A} 3$ ( $\mathrm{ABCA} 3$ ) were related to drug resistance, especially by drug expulsion which might be modulated by microparticles (Chapuy et al., 2008, Steinbach, et al., 2006). Oksvold et al. (2014) showed a similar observation that exosomes secreted by B-cell lymphoma reduced rituximab-induced cytotoxicity. These studies indicated a novel mechanism of drug resistance in lymphoma, which is linked to an ABCA3-dependent pathway of exosome secretion, (Oksvold et al., 2014). In particular, it was suggested that increased expression of cellular galectin-3 and elevated concentration of galectin-3 in circulating system may contribute to tumor progression and drug resistance. Regarding ALL, the levels of exosomal Gal-3 mRNA and protein, which originated from stromal cells in B-ALL were in relation with the appearance of drug-resistance through activated MEK/ERK pathway, (Fortuna et al., 2014). Galectin-3 is enriched in OP9 exosomes, and exosomal galectin-3 can be internalized by ALL cells, and activates $\mathrm{NF \kappa B}$ signaling pathway, whichisoften linked to anti-apoptosis and drug resistance (Hu et al., 2015). 
Another study showed that exosomal evasion of humoral immunotherapy in aggressive B-cell lymphoma wasmodulated by ATP-binding cassette transporter A3. B-cell lymphoma cells released exosomes that carried $\mathrm{CD} 20$, bound therapeutic anti-CD20 antibodies, consumed complement, thereby impairing antibodydependent cell mediated cytotoxicity (ADCC) and protected target cells from antibody attack through linked in an ABCA3-dependent pathway of exosome secretion. Additionally, removing exosomes from plasma samples of B-NHL resulted in considerable improvements in the effect of rituximab against lymphoma cell in vitro, (Aung et al., 2011). In multiple myeloma, the integrinmediated interaction of cancer cells with MSC, upregulates the secretion of soluble factors such as IGF-1, FGF, IL-6 and others that provide growth advantage and drug resistance to multiple myeloma cells (Isidori et al., 2014, Greco et al., 2009).

Another study by Wang et al. (2014) indicated that bone marrow stromal cell-derived exosomes in MM can promote proliferation, migration, and survival of MM cells, andcan also induce chemotherapy drug resistance to bortezomib, influencing several pathways including c-Jun N-terminal kinase, p38, p53, and Akt which are relevant to survival of MM cells.All these studies suggest that exosome mediate drug resistance through both the direct shuttling of drugs out of the cells and the horizontal transfer of molecules/molecular signals that bestow drug resistance to the otherwise sensitive cells.

Immune escape: Hematological malignancy cells can evade host immune surveillance, a well-known phenomenon called as immune evasion or immune escape, which is also a hallmark of tumor. Many research groups have demonstrated abundant quantity of exosomes released by tumor cells exerting an immunosuppressive effect that helps them evade immune response. Dysfunctions of natural killer (NK) cells, which are a major component of the anti-tumor immune response, have been reported in various hematologic malignancies, including chronic lymphocytic leukemia (CLL). BAG6, which issecreted by tumor cell, activate receptor NKp30 on the surface of NK cells, further increase NK cell cytotoxicity and promote NK cells to kill cancer cells, and is suggested that downregulated or absent of exosomal BAG6 in CLL patients evasion of CLL cells from NK cell anti-tumor activity, (Reiners et al., 2013).

TGF- $\beta 1$ is a potent immunosuppressive molecule that inhibits NK cell cytotoxicity. The serum concentration of exsomal TGF- $\beta 1$ in the newly diagnosed AMLwassignificantly higher than that of the normal control. Furthermore, NK cell differentiation is IL-15 dependent, and IL-15 plays a major role in NK-cell expansion and promotes NK-cell survival, IL-15 is also able to counteract immunosuppressive effects mediated by TGF- $\beta$ car- ried on exosomes from AML patients. Taken together, these evidence suggest IL-15 can enhance anti-tumor effects of NK cells in AML patients, (Greco et al., 2009, Hong et al., 2014). In virus-related lymphomas, EBV+ lymphoma cells are embedded in non-neoplastic by standers: B and T cells, macrophages. There is increasing evidence that the indirect actions (i.e. immunosuppression and TME components) of different viruses also play significant roles in lymphomagenesis, (Esau, 2017).

For example, EBV and HIV-1 are proved capable of inducing the overexpression of PD-L1 on antigenpresenting cells, thus resulting in immunosuppression by the increased apoptosis of T cells. Furthermore, the EBV-miRNAs in the exosome secreted from EBV positive lymphoma cells were transferred to macrophage and promote the development of lymphoproliferative disease (LPD) in vivo mouse model, (Lichterfeld et al., 2008). Another study showed that the exosomes present in the serum of CHB patients contain both HBV nucleic acids and HBV proteins, HBV might influence NK-cell function via exosomes through upregulating immunosuppressive factors, such as TGF- $\beta$, in HBV-infected CHB patients. These observations suggest that exosomes may serve as important regulators of HBV transmission and may be involved in escaping innate immunity.

\section{THE ROLE OF EXOSOMES IN DIAGNOSIS AND PROGNOSIS OF HEMATOLOGICAL MALIGNANCIES}

Early cancer detection and disease stratification or classification are critical to successful treatment. Accessible, reliable, and informative hematological malignancies biomarkers can be medically valuable and can provide some relevant insights into cancer biology [Elsherbini and Bieberich., 2018]. Early detection of cancer could be easily performed using exosomes isolated from body fluids such as blood plasma, serum, and urine, which is allowing for a non-invasive method of detection of hematological malignancies, (Kang et al., 2018).

Microvesicles are found in higher concentrations in the sera of different types of hematological malignancies, such as AML, ALL, CML, CLL, diffuse large B-cell lymphoma (DLBCL), Hodgkin's lymphoma (HL) and MM patients and abundantly express surface proteins unique to their cell of origin, which is rarely observed from serum microvesicles of normal controls [Kang et al., 2018]. AML biomarkers NPM1, FLT3, CXCR4, MMP9 and IGF-IR are also present in AML cell derived exosomes, along with mRNAs involved in leukemia development(Boyiadzis, 2016). Hong. et al (2014). indicated that exosomal TGF$\beta 1$ levels and relative levels of the three TGF- $\beta 1$ forms (TGF- $\beta 1$ pro-peptide, latency-associated peptide (LAP), and mature TGF- $\beta 1$ were distinct in AML patients in 
Table 1 . The role of exosome in the development of hematologic tumor.

\begin{tabular}{|c|c|c|c|c|}
\hline Targets & Type & Disease & Function & Reference \\
\hline $\begin{array}{l}\text { TGF- } \beta 1 \text { MICA, } \\
\text { MICB,ULBP 1,ULBP2, } \\
\text { BAG6 }\end{array}$ & protein & AML & $\begin{array}{l}\text { Reduce the ability of } \\
\text { natural killer (NK) } \\
\text { cells to kill leukemic } \\
\text { cells. }\end{array}$ & $\begin{array}{l}\text { Cheng et al., } 2011 \\
\text { Jaworski et al., } 2014\end{array}$ \\
\hline $\begin{array}{l}\text { GATA1, F0X3, SHIP } 1 \text {, } \\
\text { ID } 1 \text {, E2F1, CEBP- } \alpha \text {, } \\
\text { CEBP- } \beta \text {, Myc, MEF2C }\end{array}$ & protein & AML & $\begin{array}{l}\text { Induce development } \\
\text { of leukemia. }\end{array}$ & Malla et al., 2018. \\
\hline $\begin{array}{l}\text { PI3/AKT,MAPK/ERK, } \\
\text { NF-kB,TGF- } \beta 1\end{array}$ & protein & CML & $\begin{array}{l}\text { Enhance cell survival } \\
\text { of LAMA84 cell }\end{array}$ & Sun et al., 2017 \\
\hline $\begin{array}{l}\text { Tax, AKT, Rb, cFLIP, } \\
\text { NF-kB }\end{array}$ & & ATL & $\begin{array}{l}\text { Enhance cell survival } \\
\text { in murine and human } \\
\text { T-cell cell lines. }\end{array}$ & Hong et al., 2014 \\
\hline Galectin-3, NF-kB & protein & ALL & $\begin{array}{l}\text { Promote ALL drug } \\
\text { resistance. }\end{array}$ & Hong et al., 2014 \\
\hline SRC, TSP-1, IL-8 & protein & CML & $\begin{array}{l}\text { Promotes endothelial } \\
\text { cell angiogenesis. }\end{array}$ & Nanbo et al., 2013 \\
\hline MiR-210 & microRNA & CML & $\begin{array}{l}\text { Promote the vascular } \\
\text { activity of CML. }\end{array}$ & Nanbo et al., 2013 \\
\hline Wnt3a & protein & DLBCL & $\begin{array}{l}\text { Promote the growth of } \\
\text { DLBCL cells. }\end{array}$ & Huan et al., 2014 \\
\hline $\begin{array}{l}\text { IL-6, CCL2, } \\
\text { fibronectin }\end{array}$ & protein & MM & $\begin{array}{l}\text { Promote the growth of } \\
\text { MM cells. }\end{array}$ & Raimondo et al., 2015 \\
\hline miR-135b & microRNA & MM & $\begin{array}{l}\text { Promoteendothelial } \\
\text { vessel formation. }\end{array}$ & De et al., 2016 \\
\hline $\begin{array}{l}\text { miR-34a, miR-125b- 5p, } \\
\text { miR-146a, miR-15a, miR } \\
\text {-137/197, miR- } 21\end{array}$ & microRNA & MM & $\begin{array}{l}\text { Facilitate multiple } \\
\text { myeloma growth and } \\
\text { progression }\end{array}$ & $\begin{array}{r}\text { Nanbo et al., } 2013 \\
\text { Jaworski et al., } 2014\end{array}$ \\
\hline
\end{tabular}

AML: acute myeloid leukemia; CML: chronic myelocytic leukemia; ATL: T-cell acute lymphoblastic leukemia; ALL: acute lymphoblastic leukemia; DLBCL: diffuse large B-cell lymphoma; MM: multiple myeloma

different stages of chemotherapy[Hong C.S. et al., 2014]. This stability makes exosomes as suitable mines for hunting reproducible and consistent biomarkers. ALLassociated expression of miRNA92a can be detected in the circulating vesicles of a majority of ALL patients (Rekker K. et al., 2014)

In CML, Mineo et al. (2012), showed that a co-evolution between endothelial cells and CML cells are essential Table 2. Circulating vesicles as a general hematological malignancies biomarker.

\begin{tabular}{|l|l|l|l|l|}
\hline Biomarker & Type & Source & $\begin{array}{l}\text { Hematological } \\
\text { Malignancies }\end{array}$ & Reference \\
\hline $\begin{array}{l}\text { NPM1, FLT3, CXCR4, MMP9 } \\
\text { and IGF-IR }\end{array}$ & protein & serum & AML & Wang et al., 2014 \\
\hline MiR-92a & MicroRNA & serum & ALL & Hong et al., 2014 \\
\hline TGF- $\beta 1$ & protein & serum & AML & Reiners et al., 2014 \\
\hline miR155 & MicroRNA & serum & $\begin{array}{l}\text { AML, CLL, WM, } \\
\text { MDS, MM }\end{array}$ & $\begin{array}{l}\text { Elsherbini ct } \\
\text { Bieberich 2018 }\end{array}$ \\
\hline Src & protein & serum & CML & Hong et al., 2014 \\
\hline MiR-155, MiR-210, MiR-21 & MicroRNA & serum & DLBCL & Rekker et al., 2014 \\
\hline MiR-22 & MicroRNA & plasma & HL & Caviano et al., 2014 \\
\hline MiR-15a, let-7b miR-18a, & MicroRNA & plasma & MM & Caviano et al., 2014 \\
\hline $\begin{array}{l}\text { AML: acute myeloid leukemia; ALL: acute lymphoblastic leukemia; CLL: chronic lymphocytic leukemia; WM: } \\
\text { macroglobulinemia of Waldenstrom; MDS: myelodysplastic syndrome; MM: multiple myeloma; CML: chronic } \\
\text { myelocytic leukemia; DLBCL: diffuse large B-cell lymphoma; HL: Hodgkin's lymphoma. }\end{array}$
\end{tabular}


for leukemia progression and resistance to therapy. This is possible because of the fact that K562 malignant cells secret growth factors and various miRNAs and transport these 'endothelial inducing factors' via exosomes. As a result, tube formation is stimulated, even when treating with imatinib, a tyrosine kinase inhibitor that targets the Philadelphia chromosome-positive $(\mathrm{Ph}+)$ myeloid leukemia cells. In this case, the development of angiogenesis was reported to regulate the progression and dissemination of this hematological malignancy, (Wojtuszkiewicz et al., 2016 Caivano et al., 2017).

Parikh et al. (2016) found the EV miR155 level may serve as a promising prognostic/predictive biomarker in CLL, independent of clinical stage. In conjunction with the previously reported data, Caivano et al. (2017) found that the EV miR155 levels were significantly higher in CLL, AML and Waldenström'smacroglobulinemia (WM) cases compared to controls (Parikh et al., 2016, Zhu et al., 2018). Conversely, they also found that the EV miR155 levels were significantly lower in myelodysplastic syndrome (MDS) and MM cases, (Caivano et al., 2017). In addition, they found that high EV miR155 levels correlated with high white blood cell counts in AML patients. In conclusion, this study indicated that EV miR155 may serve as an attractive new, non-invasive diagnostic biomarker in human hematologic malignancies. Levels of exosomal miR-155, miR-210 and miR-21 in serum from DLBCL patients $(\mathrm{n}=60)$ were higher than control sera $(\mathrm{n}=43)(\mathrm{P}=0.009)$, (Mineo et al. 2012):

Monique et al. found that EV-associated miR21-5p, miR127-3p, let7a-5p, miR24-3p, and miR155-5p signals were higher in primary and relapsed classic Hodgkin's lymphoma (cHL) patients compared with healthy individuals [van Eijndhoven M.A. et al., 2016]. However, they also detected that miR21-5p and miR155-5p is small, but signifcant, decreases (2-fold, $\mathrm{P}=0.016$ ) in plasma after therapy. Nevertheless, the decrease in miR155-5p was more pronounced in the EV fraction, (4-fold, $\mathrm{P}=$ 0.016). They detected high levels of miR127-3p in EVs produced by HRS cells in plasma EVs of cHL patients but less abundant levels in healthy control EVs, which suggests that the pool of miR127-3p detected in the protein fraction is unrelated to cHL tumor tissue and is derived from other sources [van Eijndhoven M.A., et al., 2016]. miR-15a is lower in mesenchymal stromal cells derived vesicles of MM patients compared with healthy subject, and miRNA-rich exosomes secreted from MM-mesenchymal stromal cells facilitate MM progression, (Manier et al., 2017).

Lower expression of let-7b or miR-18a was significantly associated with a high ISS stage. However, both let-7b and miR-18a were independent predictors after adjusting for the ISS and specific cytogenetic abnormalities. The effect of the two miRNAs on PFS and OS was illustrated by Kaplan-Meier curves with dichotomized miRNAs at the median [Manier S. et al., 2017]. These data indicate that specific miRNAs can be critical in defining worse prognosis in patients with newly diagnosed MM. Nevertheless, to establish circulating exosomes as biomarkers, well-designed clinical trials are required. So far, there is no trial registered that is relevant to hematology and investigates circulating exosomes as a predictive marker in hematological malignancies. Exosomes are currently viewed as tumor cell surrogates or 'liquid biopsies' and as a promising non-invasive metrics of cancer. Exosomes might emerge as the most informative non-invasive predictors of cancer outcome orresponse to therapy.

\section{EXOSOME-BASED HEMATOLOGIC MALIGNANCIESTHERAPEUTICS}

Exosome-based therapies serve as attractive strategy against hematologic malignancies and solid tumors. Exosome-based delivery methods have been tested in the clinic successfully and were found to be well tolerated in patients. Being autologously generated within the host, they can be engineered to carry drugs or target proteins without invoking immunogenic response. A number of different strategies have been applied to harness the potential of these exosomes. Nano injections of RNAi in dendritic-derived exosomes allowed delivery to the brain without invoking immune response. These findings were confirmed when Gurwitz et al. showed that siRNAs can be delivered across the blood brain barrier in a mouse model using systemic injections of exosomes, (Gurwitz, 2016).

In another study, tumor-derived exosome-pulsed DCs, tumor-derived exosomes, and exosomes isolated from malignant ascites all have been investigated for their ability to elicit antitumor immune response in patients, (Liu et al., 2018). Likewise, their efficacy on exosome release or against exosomes has also been tested in different laboratories independently. These are some of the studies highlighting the benefit of applying nanobased assays in the design of exosomes drug therapies for cancer. However, its clinical utility needs to be tested in future studies. At present, there are several clinical studies that utilize an exosome-based regimen for solid tumors, (Que et al., 2016), but there is no clinical trial about exosome-based agents against hematologic malignancies (ClinicalTrials.gov Website keyword search exosome). These studies clearly point to an unexplored area of research where researchers can find answers to some of the unexplained mechanisms attributed to the multifaceted natural agents against hematologic malignancies. Altogether, exosomes have multiple potential clinical uses including the development of vaccines for 
targeting tumors; also, tumor-derived exosomes may be useful as surrogate endpoints in evaluating therapeutic and preventive approaches to hematological tumors.

\section{CONCLUSIONS}

Success in diagnosis and treatment of complex hematological malignancies is dependent on our full understanding of the intricacies of interactions between different components within tumors. On the one hand, a number of studies showed exosomes are emerging as major players in inter- and intracellular communications. Exosomes have been shown to secrete diverse biological molecules, which are in the context of tumor cells survival, metastasis, drug resistance and immune evasion. On the other hand, tumor relevant exosomes play the important role in the areas of diagnostics and drug therapy, regenerative medicine, and vaccines. Taken together, technology and biology will inevitably pave the way for the future use of exosomes analysis in many preclinical research and clinical applications.

\section{FUNDING}

This work was supported by grants 81600163 and 81570201 from the National Natural Science Foundation of China (NSFC) and Shandong Provincial Natural Science Foundation (ZR2017PH057).

\section{REFERENCES}

Kornblau SM, et al. (1998). The role of apoptosis in the pathogenesis, prognosis, and therapy of hematologic malignancies. Leukemia, 12(1), 41-6.

Li, X, H. Zhong, et al. (2016). The diagnosis, prognosis, and therapeutic application of MicroRNAs in haematological malignancies. Hematology, 21(5): p. 263-71.

Rama Rao Malla, Santhi Pandrangi, Seema Kumari, et al. (2018), Exosomal tetraspanins as regulators of cancer progression and metastasis and novel diagnostic markers. Asia Pac J Clin Oncol, DOI: 10.1111/ajco.12869.

Pfrieger, F.W. and N. Vitale. (2018), Cholesterol and the journey of extracellular vesicles. Journal of lipid research, 59(9).

Whiteside, T.L., M. Boyiadzis, (2017), Response commentary: exosomes vs microvesicles in hematological malignancies. Leukemia, 31(10): p. 2277.

Sun Z, S Yang, Q Zhou, et al. (2018), Emerging role of exosome-derived long non-coding RNAs in tumor microenvironment. Mol Cancer, 17(1): p. 82.

Hong, C.S., et al. (2014), Plasma exosomes as markers of therapeutic response in patients with acute myeloid leukemia. Front Immunol, 5: 160.

Huan, J., et al. (2013), RNA trafficking by acute myelogenous leukemia exosomes. Cancer Res, 73(2):. 918-29.
Huan, J.,et al. (2013), RNA trafficking by acute myelogenous leukemia exosomes. Cancer Res, 73(2): 918-29.

Long, Q., et al. (2017), Intranasal MSC-derived A1-exosomes ease inflammation, and prevent abnormal neurogenesis and memory dysfunction after status epilepticus. Proc Natl Acad Sci U S A,114(17): E3536-E3545.

Zhou, J., et al. (2012), The pro-metastasis tyrosine phosphatase, PRL-3 (PTP4A3), is a novel mediator of oncogenic function of BCR-ABL in human chronic myeloid leukemia. Mol Cancer, 11: 72.

Raimondo, S., et al. (2015), Chronic myeloid leukemia-derived exosomes promote tumor growth through an autocrine mechanism. Cell Commun Signal, 13:8.

Jaworski, E., et al. (2014), Human T-lymphotropic virus type 1-infected cells secrete exosomes that contain Tax protein. J Biol Chem, 289(32): 22284-305.

Koch, R., et al. (2014), Populational equilibrium through exosome-mediated Wnt signaling in tumor progression of diffuse large B-cell lymphoma. Blood, 123(14): 2189-98.

Hazan-Halevy, I., et al. (2015), Cell-specific uptake of mantle cell lymphoma-derived exosomes by malignant and nonmalignant B-lymphocytes. Cancer Lett, 364(1): 59-69.

Nanbo, A., et al. (2013), Exosomes derived from Epstein-Barr virus-infected cells are internalized via caveola-dependent endocytosis and promote phenotypic modulation in target cells. J Virol, 87(18): 10334-47.

Chugh, P.E., et al. (2013), Systemically circulating viral and tumor-derived microRNAs in KSHV-associated malignancies. PLoS Pathog, 9(7): e1003484.

Roccaro, A.M., et al. (2013), BM mesenchymal stromal cellderived exosomes facilitate multiple myeloma progression. J Clin Invest, 123(4): 1542-55.

De Veirman, K., et al. (2016), Induction of miR-146a by multiple myeloma cells in mesenchymal stromal cells stimulates their pro-tumoral activity. Cancer Lett, 377(1): 17-24.

Umezu, T., et al. (2013), Leukemia cell to endothelial cell communication via exosomal miRNAs. Oncogene, 32(22): 274755.

Fang, Y., et al. (2016), PML-RARa modulates the vascular signature of extracellular vesicles released by acute promyelocytic leukemia cells. Angiogenesis, 19(1): 25-38.

Tadokoro, H., et al. (2013), Exosomes derived from hypoxic leukemia cells enhance tube formation in endothelial cells. J Biol Chem, 288(48): 34343-51.

Paggetti, J., et al. (2015), Exosomes released by chronic lymphocytic leukemia cells induce the transition of stromal cells into cancer-associated fibroblasts. Blood, 126(9): 1106-17.

Yoon, C., et al. (2016), Delivery of miR-155 to retinal pigment epithelial cells mediated by Burkitt's lymphoma exosomes. Tumour Biol, 37(1): 313-21.

Umezu, T., et al. (2014), Exosomal miR-135b shed from hypoxic multiple myeloma cells enhances angiogenesis by targeting factor-inhibiting HIF-1. Blood, 124(25): 3748-57. 
Rizzo, M.T., et al. (2011), Cyclooxygenase-2 in oncogenesis. Clin Chim Acta, 412(9-10): 671-87.

Isidori, A., et al. (2014), The role of the immunosuppressive microenvironment in acute myeloid leukemia development and treatment. Expert Rev Hematol, 7(6): 807-18.

Viola, S., et al. (2016), Alterations in acute myeloid leukaemia bone marrow stromal cell exosome content coincide with gains in tyrosine kinase inhibitor resistance. Br J Haematol, 172(6): 983-6.

Cheng, Y.L., et al. (2011), Increased galectin-3 facilitates leukemia cell survival from apoptotic stimuli. Biochem Biophys Res Commun, 412(2): 334-40.

$\mathrm{Hu}$, K., et al. (2015), Galectin-3 mediates bone marrow microenvironment-induced drug resistance in acute leukemia cells via Wnt/beta-catenin signaling pathway. J Hematol Oncol, 8:1.

Fortuna-Costa, A., et al. (2014), Extracellular galectin-3 in tumor progression and metastasis. Front Oncol, 4: 138.

Chapuy, B., et al. (2008), Intracellular ABC transporter A3 confers multidrug resistance in leukemia cells by lysosomal drug sequestration. Leukemia, 22(8): 1576-86.

Steinbach, D., et al. (2006), ABCA3 as a possible cause of drug resistance in childhood acute myeloid leukemia. Clin Cancer Res, 2006. 12(14): 4357-63.

Oksvold, M.P., et al. (2014), Expression of B-cell surface antigens in subpopulations of exosomes released from B-cell lymphoma cells. Clin Ther, 36(6): 847-862.

Aung, T., et al. (2011), Exosomal evasion of humoral immunotherapy in aggressive B-cell lymphoma modulated by ATPbinding cassette transporter A3. Proc Natl Acad Sci U S A, 108(37): 15336-41.

Greco, C., et al. (2009), Reduction of serum IGF-I levels in patients affected with Monoclonal Gammopathies of undetermined significance or Multiple Myeloma. Comparison with bFGF, VEGF and K-ras gene mutation. J Exp Clin Cancer Res, 28: 3

Wang, J., et al. (2014), Bone marrow stromal cell-derived exosomes as communicators in drug resistance in multiple myeloma cells. Blood, 124(4): 555-66.

Reiners, K.S., et al. (2013), Soluble ligands for NK cell receptors promote evasion of chronic lymphocytic leukemia cells from NK cell anti-tumor activity. Blood, 121(18): 3658-65.

Hong, C.S., et al. (2014), Plasma exosomes as markers of therapeutic response in patients with acute myeloid leukemia. Front Immunol, 5: 160.

Esau, D. (2017), Viral Causes of Lymphoma: The History of Epstein-Barr Virus and Human T-Lymphotropic Virus 1. Virology (Auckl), 8: 1178122X17731772.

Lichterfeld, M., et al. (2008), Telomerase activity of HIV-1-specific CD8+ T cells: constitutive up-regulation in controllers and selective increase by blockade of PD ligand 1 in progressors. Blood, 112(9): 3679-87.
Elsherbini, A., E. Bieberich. (2018), Ceramide and Exosomes: A Novel Target in Cancer Biology and Therapy. Adv Cancer Res, 140: 121-154.

Kang, K.W., et al. (2018), The Potential of Exosomes Derived from Chronic Myelogenous Leukaemia Cells as a Biomarker. Anticancer Res, 38(7): 3935-3942.

Boyiadzis, M.,T.L. (2016), Whiteside, Plasma-derived exosomes in acute myeloid leukemia for detection of minimal residual disease: are we ready? Expert Rev Mol Diagn, 16(6): 623-9.

Hong, C.S., et al. (2014), Plasma exosomes as markers of therapeutic response in patients with acute myeloid leukemia. Front Immunol, 5: 160.

Rekker, K., et al. (2014), Comparison of serum exosome isolation methods for microRNA profiling. Clin Biochem, 47(1-2): 135-8.

Mineo, M., SH Garfield, S Taverna, et al. (2012), Exosomes released by K562 chronic myeloid leukemia cells promote angiogenesis in a Src-dependent fashion. Angiogenesis, 15(1), 33-45.

Caivano, A., et al. (2017), MicroRNA-155 in serum-derived extracellular vesicles as a potential biomarker for hematologic malignancies - a short report. Cell Oncol (Dordr), 40(1): 97-103.

Wojtuszkiewicz, A., et al. (2016), Exosomes Secreted by Apoptosis-Resistant Acute Myeloid Leukemia (AML) Blasts Harbor Regulatory Network Proteins Potentially Involved in Antagonism of Apoptosis. Mol Cell Proteomics, 15(4): 1281-98.

Parikh, S.A., T.D. (2016), Shanafelt, Prognostic factors and risk stratification in chronic lymphocytic leukemia. Semin Oncol, 43(2): 233-40.

Zhu, H.Y., et al. (2018), [Prognostic significance of CLL-IPI for Chinese patients with chronic lymphocytic leukemia]. Zhonghua Xue Ye Xue Za Zhi, 39(5): 392-397.

Caivano, A., et al. (2017), MicroRNA-155 in serum-derived extracellular vesicles as a potential biomarker for hematologic malignancies - a short report. Cell Oncol (Dordr), 40(1): 97-103.

Mineo, M., et al. (2012), Exosomes released by K562 chronic myeloid leukemia cells promote angiogenesis in a Src-dependent fashion. Angiogenesis, 15(1): 33-45.

van Eijndhoven, M.A., et al. (2016), Plasma vesicle miRNAs for therapy response monitoring in Hodgkin lymphoma patients. JCI Insight, 1(19): e89631.

Manier, S., et al. (2017), Prognostic role of circulating exosomal miRNAs in multiple myeloma. Blood, 129(17): 2429-2436.

Gurwitz, D. (2016), MicroRNAs as CNS Drug Targets. Drug Dev Res, 77(7): 331-335.

Liu, H., et al. (2018), Dendritic cells loaded with tumor derived exosomes for cancer immunotherapy. Oncotarget, 9(2): 28872894.

Que, R.S., et al. (2016), Increasing the immune activity of exosomes: the effect of miRNA-depleted exosome proteins on activating dendritic cell/cytokine-induced killer cells against pancreatic cancer. J Zhejiang Univ Sci B, 17(5): 352-60. 